\title{
Revisión
}

\section{Uso de la metformina en pacientes con el síndrome del ovario poliquístico}

\author{
Donald Fernández-Morales, Felipe Sagot-Verdesia
}

\section{Resumen}

La metformina es un medicamento indicado para el tratamiento de la diabetes mellitus tipo II con una función renal normal. Ha sido usada, sin la aprobación de la Administración de Drogas y Alimentos de los EEUU, en pacientes con el síndrome del ovario poliquístico, como regulador del trastorno menstrual, tratamiento del hirsutismo e inductor de ovulación.

Desde 1980, Burghen y colaboradores señalaron la asociación entre el hiperandrogenismo y la hiperinsulinemia en el síndrome del ovario poliquístico. El exceso de andrógenos, resultado del aumento de la insulina, deteriora la acción de esta.

Los efectos de la metformina en la disminución de la glucosa son consecuencia de una reducción en la producción de glucosa hepática y un aumento en su empleo. Este medicamento incrementa la mayoría de las acciones biológicas de la insulina en personas con resistencia a la insulina preexistente y disminuye la absorción intestinal de glucosa.

Se concluye que la metformina es útil en la regulación el ciclo menstrual en comparación con el placebo; no es un tratamiento de primera elección en el hirsutismo y su uso como monoterapia en la inducción de la ovulación no demuestra sustancialmente mejoría en la tasa de embarazo clínico. La evidencia señala que la metformina asociada con el citrato de clomifeno induce la ovulación y mejora la tasa de embarazo clínico.

Descriptores: Metformina, síndrome del ovario poliquístico, hiperandrogenismo, hiperinsulinemia, resistencia a la insulina, acidosis láctica, oligoamenorreas, hirsutismo, inducción de ovulación

Servicio de Ginecología y Obstetricia. Hospital México Abreviaturas: IMC, indice de masa corporal; $\mathrm{Mg}$, miligramos; OR, razón de ocurrencia; RR, riesgo relativo; SOP, síndrome del ovario poliquístico.

Correspondencia: Donald Fernández Morales Apartado postal I077- 101 I Y Griega, San José Felipe Sagot Verdesia Apartado postal 136-4006 Los Arcos, Heredia

ISSN 0001-6002/2007/49/3/140-146 Acta Médica Costarricense, $\mathbb{C} 2007$ Colegio de Médicos y Cirujanos
Key words: Metformin, polycystic ovary syndrome, hyperandrogenism, hyperinsulinism, insulin resistance, lactic acidosis, oligomenorrhea, hirsutism, ovulation inductor.

Recibido: 3 de enero de 2007

Aceptado: 20 de febrero de 2007

La paciente ideal para prescripción de metformina es una persona obesa con diabetes mellitus tipo 2, con una función renal normal documentada por una concentración de creatinina $<1.4$ $\mathrm{mg} / \mathrm{dl}$, sin síntomas coexistentes de insuficiencia cardiaca congestiva o condición hipóxica respiratoria. Su uso ha demostrado ser tan efectivo como la insulina o las sulfonilureas, cuando se receta como monoterapia o se combina, y su eficacia es independiente de la edad, peso, etnia, duración de la enfermedad y niveles de insulina o péptido C. ${ }^{1}$

La metformina, en calidad de medicamento sensibilizador a la insulina, ha sido utilizada como tratamiento en el síndrome de ovario poliquístico (SOP). Cuando se administra a pacientes con resistencia a la insulina, actúa aumentado la respuesta del tejido blanco a la insulina y reduciendo la necesidad de una hiperinsulinemia compensadora. 
Las indicaciones de este medicamento se han incrementado en los últimos años, conforme se publican más artículos sobre su uso. El objetivo de esta revisión es dar respuesta a las siguientes interrogantes en pacientes con SOP: 1- ¿Puede la metformina ser útil para regular el ritmo menstrual y tratar el hirsutismo? 2- ¿La metformina sola produce ovulación y aumenta la tasa de fertilidad o es necesario que se asocie con el citrato de clomifeno?

\section{Resistencia a la insulina}

Burghen, Givens y Kitabchi, en 1980, señalaron que existe una relación entre el hiperandrogenismo y el hiperinsulinismo en pacientes obesas con SOP. Su estudio de casos y controles en 14 mujeres mostró una correlación entre los niveles de testosterona e insulina basal y la respuesta de insulina a la glucosa oral. ${ }^{2}$ Dunaif refiere que el SOP se asocia con disminución de la sensibilidad a la insulina en pacientes obesas y delgadas. ${ }^{3}$ Sin embargo, Holte menciona que la diferencia del índice de resistencia a la insulina es más significativa si el IMC es mayor, siendo determinada por la distribución de la grasa corporal. ${ }^{4}$ Cibula comenta que la evidencia de la resistencia a la insulina se basa en grupos pequeños de pacientes examinados y que probablemente existe diferencia en la disminución de la sensibilidad en diversos subgrupos de pacientes con SOP. ${ }^{5}$

No hay ningún examen clínico validado para detectar la resistencia a la insulina en la población general. ${ }^{6,7}$ Se han desarrollado diversos criterios para definir un síndrome metabólico, que incluyen componentes asociados con la resistencia a la insulina, como obesidad centrípeta, hipertensión arterial, alteración de la glicemia en ayunas y dislipidemia, ${ }^{6}$ aunque su etiología permanece incierta. ${ }^{8}$

Se han usado otros métodos como la pérdida de peso, nutrición adecuada y ejercicio, con el fin de disminuir el hiperinsulinismo, pero el grado de cumplimiento ha sido pobre y los beneficios de la disminución de peso no se mantienen. Dada la fuerte evidencia de que la hiperinsulinemia desempeña un papel importante en el desarrollo del SOP, es razonable asumir que las intervenciones que reduzcan los niveles circulantes de insulina, restauran la función reproductiva normal. ${ }^{9}$

\section{Farmacología}

La metformina, una biguanida de segunda generación, fue introducida al mercado de Estados Unidos en 1995. Este medicamento ha sido aprobado por la Administración de Drogas y Alimentos para el manejo de la diabetes mellitus tipo 2, cuando no se obtiene un control adecuado de la glicemia con dieta y ejercicio. Puede usarse concomitantemente con sulfonilurea o insulina, o como monoterapia. ${ }^{10}$

Sus efectos de disminución de la glucosa son principalmente consecuencia de una reducción en la producción de glucosa hepática y un aumento en su utilización, estimulada por la insulina, en células esqueléticas $\mathrm{y}$ adipositos. La merma en la producción de glucosa se atribuye a la reducción en la gluconeogénesis. Los efectos de la metformina en tejidos periféricos sensibilizados a la insulina requieren la presencia de esta. El medicamento incrementa la mayoría de las acciones biológicas de la insulina, incluyendo el transporte de glucosa y la síntesis de glicógeno y lípidos, en personas con resistencia a la insulina preexistente. También disminuye la absorción intestinal de glucosa e, indirectamente, aumenta la respuesta de las células $\mathrm{B}$ pancreáticas al reducir la oxidación de ácidos grasos libres. ${ }^{1}$

El hiperinsulismo, como reflejo de la resistencia a la insulina, contribuye a la excesiva producción de testosterona por los ovarios y las adrenales, disminuyendo la producción de la globulina hepática transportadora de hormona sexual e incrementando los niveles de testosterona libre y total. ${ }^{11} \mathrm{El}$ exceso de andrógenos deteriora la acción de la insulina creándose un círculo vicioso cuyo inicio es difícil identificar. La metformina, al incrementar la sensibilidad a la insulina y disminuir sus niveles, reduce la producción de andrógenos y aumenta el estradiol en el SOP. ${ }^{1}$

La metformina es rápidamente absorbida del intestino delgado, y alcanza niveles elevados a las dos horas de su ingestión. $\mathrm{Su}$ excreción es por orina, un 90\% como medicamento sin cambio, posiblemente por secreción tubular. Algunos medicamentos, como cimetidina $\mathrm{y}$ furosemida, incrementan su concentración plasmática, y otros, como digoxina, morfina, ranitidina, trimetoprim y vancomicina, pueden competir por la vía renal de excreción. $^{12}$

Se presenta en tabletas de clorhidrato de metformina de 500,850 y $1000 \mathrm{mg}$. La posología promedio en mayores de 17 años es de 500 a $850 \mathrm{mg}$ por la mañana, después del desayuno, y 500 a $850 \mathrm{mg}$ por la noche, después de la cena, ingiriendo cada tableta en forma íntegra. La dosis se puede incrementar en una tableta cada 1 ó 2 semanas, hasta un máximo de $2500 \mathrm{mg}$ por día. ${ }^{1,9}$

\section{Efectos secundarios}

Las reacciones adversas más frecuentemente mencionadas son: náuseas y vómitos en un $6-25 \%$, diarrea en un 10 - 53\%, flatulencia en un $12 \%$, disminución de vitamina B12 en un 7\% y cefalea en un $6 \%{ }^{13}$

La tasa de incidencia de acidosis láctica es de 5 por $100.000 .{ }^{14}$ Se ha reconocido durante años que la terapia con biguanidas se asocia con acidosis láctica, condición relacionada con alta mortalidad (30-50\%). Sin embargo, las determinaciones de los niveles de ácido láctico arterial no han tenido valor pronóstico, y altas concentraciones de metformina han sido asociadas con mejor sobrevivencia. Se estima que el determinante del pronóstico de sobrevivida de cada paciente es la enfermedad concurrente. ${ }^{15}$ 
Las condiciones médicas que precipitan la acidosis láctica en pacientes tratados con metformina son las siguientes: insuficiencia renal en la mayoría de los casos, insuficiencia cardiovascular, sepsis, hemorragia, insuficiencia hepática o pulmonar; otra contraindicación para su uso es historia previa de acidosis láctica. Dicho medicamento debe suspenderse temporalmente antes de cualquier procedimiento de cirugía mayor que obligue a restringir la ingesta de líquidos.

Calabrese y otros identificaron 263 hospitalizaciones de 204 pacientes que recibieron al menos una dosis de metformina, quienes mostraban una contraindicación absoluta para su uso. Los niveles elevados de creatinina estaban presentes en 32 admisiones, aunque la metformina fue descontinuada en 8 pacientes. Este estudio demuestra la importancia de que los profesionales en medicina conozcan mejor las directrices para prescribir tal medicamento. ${ }^{16}$

\section{Indicaciones}

Estudios han evidenciado que la metformina puede usarse para el tratamiento de ciertos rasgos del SOP, como el hiperandrogenismo, las irregularidades menstruales, la resistencia a la insulina, la disminución de ovulación y la infertilidad. Sus efectos son probablemente mediados por una reducción de los niveles de insulina, que interfiere con la producción de andrógenos. Pareciera lógico tratar la causa, resistencia a la insulina, y no el síndrome resultante. En teoría, al incrementar la sensibilidad a la insulina, se pueden beneficiar otros aspectos del SOP.

\section{Oligoamenorrea}

Costello y Eden refieren que existen, hasta junio de 2002, 12 estudios que evalúan la efectividad de la monoterapia con metformina en la restauración de menstruaciones regulares en pacientes con SOP. El total de pacientes en estos estudios es de 300 , pero en ninguno de ellos el número es mayor de 50. Nueve de las investigaciones fueron observacionales y no controladas y solo 3 son aleatorias. Al combinar los resultados de los estudios observacionales se demuestra que la metformina restaura las menstruaciones regulares en un $62 \%$ de las pacientes con SOP y oligoamenorrea o amenorrea. ${ }^{17}$

Harborne y colaboradores señalan que la metformina sola no restaura el ritmo menstrual cíclico en corto tiempo en pacientes con SOP. El resultado de una función ovárica normal ocurre en menos del 50\%, con metformina en dosis usuales. Sin embargo, el uso de contraceptivos orales combinados, la mejor manera de lograr ciclos menstruales regulares, puede ser controversial en mujeres con IMC $>30$ Kg. $/ \mathrm{m}^{2}$, y exacerbar el estado de resistencia a la insulina. ${ }^{18}$

Kashyap y otros refieren, en su revisión sistemática sobre el uso de agentes insulina sensibles como terapia primaria en pacientes con SOP, que la metformina es beneficiosa para regular ciclos menstruales, en comparación con el placebo (RR 1.45, rango de confianza al 95\%, de 1.11 a 1.90).19

\section{Hirsutismo}

Crave y otros confirmaron el efecto producido por la pérdida de peso en la disminución del hiperinsulinismo e hiperandrogenismo en 24 pacientes obesas e hirsutas. Su estudio aleatorio y doble enmascarado, identificó por ultrasonido la morfología de ovarios poliquísticos en 8 pacientes del grupo y $7 \mathrm{del}$ control. ${ }^{20}$ Todas recibieron una dieta baja en calorías (1500 por día) y después fueron asignadas aleatoriamente a recibir metformina $(1700 \mathrm{mg}$ por día) o placebo por 4 meses. Los resultados mostraron que el puntaje del hirsutismo no mejoró después de 4 meses de tratamiento. No hubo diferencia significativa en insulina, globulina transportadora de hormona sexual o andrógenos entre el grupo de estudio y el de control. Los autores no demostraron beneficio en la administración de metformina como tratamiento del hirsutismo.

Kolodziejczyk y colaboradores evalúan el efecto de la metformina, en una dosis de $500 \mathrm{mg}$ tid por 12 semanas, en índices clínicos y hormonales en pacientes con SOP. Su estudio prospectivo en 34 pacientes demuestra una disminución de la testosterona total y un aumento de la globulina transportadora de hormona sexual, reduciéndose el índice de testosterona libre. El acné y la longitud del ciclo menstrual mejoran con la metformina. ${ }^{21}$

Elter, Imir y Durmusoglu designaron un estudio aleatorio para comparar los efectos endocrinos, clínicos y metabólicos de etinil estradiol y acetato de ciproterona versus metformina asociada al anterior, en pacientes no obesas con SOP. Las 40 pacientes fueron reevaluadas tras un seguimiento de 4 meses, y se encontró una disminución significativa de globulina transportadora de hormona sexual en el grupo con metformina asociado al contraceptivo oral, aunque no se afectaron los niveles de testosterona libre. ${ }^{22}$

Harborne y otros evalúan si la metformina tiene algún efecto en el hirsutismo de mujeres con SOP y su eficacia en comparación con un medicamento establecido en su tratamiento, combinando etinil estradiol y acetato de ciproterona. $\mathrm{Su}$ estudio aleatorizado por tabla de randomización de 52 pacientes mostró en ambos grupos una reducción del índice de Ferriman Gallwey (puntaje que valora el grado de hirsutismo), andrógenos totales y libres a los 12 meses. $^{23}$

Rosa Maciel y colaboradores investigan en un estudio aleatorio, enmascarado y controlado con placebo, si la metformina en dosis de $1.500 \mathrm{mg}$ /día por 6 meses, puede disminuir el hiperandrogenismo en pacientes con el SOP obesas y no obesas. De las 29 pacientes estudiadas, el grupo en tratamiento de no obesas con metformina mostró una disminución en la concentración de insulina en ayunas, testosterona total, testosterona libre y androstenediona. Sin embargo, el número de pacientes es pequeño y no refiere los rangos de confianza de los resultados. ${ }^{24}$ 
Cibula y otros publican un estudio aleatorio, no enmascarado, en 28 pacientes con el SOP, evaluando el efecto del tratamiento con un anticonceptivo oral solo, versus combinado con metformina, en 6 meses. No hubo diferencia significativa en el índice de sensibilidad a la insulina documentado por la prueba de la abrazadera euglicémica. En ambos grupos se demostró una disminución de los andrógenos y la hormona transportadora de hormona sexual, aunque el índice de andrógenos libres fue significativamente mayor en el grupo estudio, pero con rangos de confianza del $95 \%$ muy amplios (-13.0 a 5.0 vs. -21.1 a 10.0$).^{25}$

Harborne señala en su revisión, que 6 ensayos han examinado el tratamiento de hirsutismo hasta 2002, con dosis de metformina que varían de 1.500 a $1.700 \mathrm{mg}$ por día. En 3 de los estudios no hubo cambio y 3 mostraron reducción significativa en el índice de Ferriman - Gallwey. La mayoría de las investigaciones tuvieron una duración de 3 a 6 meses e incluyeron un pequeño número de pacientes. 18 Este autor recuerda que cualquier respuesta al tratamiento se debe valorar a los 6 meses, debido al promedio del crecimiento del vello.

McCarthy y otros relatan que 5 estudios aleatorios y controlados con placebo documentan una reducción significativa en la testosterona libre con metformina. Los efectos de la metformina comparados con placebo son mínimos en la testosterona total. Los estudios observacionales con 85 pacientes también muestran disminución de los niveles de andrógenos después de tratamiento con metformina. Sin embargo, a pesar de una aparente normalización bioquímica, la mejora clínica del hirsutismo no ha sido observada de manera confiable. ${ }^{26}$

Se conoce que la normalización del peso disminuye la resistencia a la insulina y mejora el ritmo menstrual. Un estudio aleatorio de 4 meses que compara la metformina versus el placebo demostró que la pérdida de peso disminuye el hiperandrogenismo en ambos grupos, sin beneficio adicional de la metformina en mejorar los niveles de andrógenos. ${ }^{20}$

\section{Inducción de ovulación}

Nestler y colaboradores mencionan en su estudio que su objetivo es determinar si la disminución a la resistencia de la insulina con metformina produce un aumento de la respuesta ovulatoria con clomifeno en mujeres obesas con SOP. ${ }^{27}$ Aunque no se sabe el método de aleatorización usado, 35 pacientes reciben $500 \mathrm{mg}$ tid de metformina y 26 en el grupo de control. Del grupo estudio se documentó ovulación por determinación de progesterona sérica $>8 \mathrm{ng} / \mathrm{ml}$ en $12(34 \%)$, versus 1 en el grupo de control (4\%). A 21 pacientes en el grupo de metformina y 25 en el de control se les agregó clomifeno porque no ovularon espontáneamente en la primera fase del estudio. En el grupo de estudio se produjo una disminución en el área bajo la curva de insulina, luego de la administración de glucosa oral, sin cambio en el grupo de control. Hubo ovulación en 19 pacientes del grupo de estudio (90\%) versus $2(8 \%)$ en el de control. La conclusión de los autores es que sus hallazgos evidencian que la disminución de la secreción de insulina facilita la ovulación espontánea y la inducción de ovulación con clomifeno. Es importante señalar que el tratamiento no fue enmascarado para los sujetos de estudio ni para los investigadores.

Batukan y Baysal evalúan el efecto promotor de la fertilidad de la metformina en pacientes con SOP. Su investigación prospectiva involucra a 29 pacientes infértiles con el diagnóstico de SOP, según los criterios del Instituto Nacional de Salud, y 15 mujeres con menstruaciones normales como controles. Todas las pacientes con SOP recibieron 78 ciclos de clomifeno, encontrándose ovulación en el $28.2 \%$, y una mujer resultó embarazada. ${ }^{28}$ Las pacientes que no lograron embarazo recibieron metformina $1500 \mathrm{mg}$ por día, dividida en 3 dosis, asociada con clomifeno. La ovulación en este grupo se logró en un 57.9\% (OR 0.286, con límites de confianza al $95 \%$, de 0.15 a 0.54 ) y embarazo en un $65.2 \%$ (OR 0.032 , con límites de confianza al 95\%, de 0.002 a 0.204$)$.

Hung Yu Ng y colaboradores investigaron en 20 mujeres chinas, la tasa de ovulación y los cambios hormonales y metabólicos en pacientes con SOP y resistentes al citrato de clomifeno, con el uso de metformina durante 3 meses. ${ }^{29} \mathrm{El}$ estudio aleatorio por computadora, doble ciego, con grupos de estudio y control comparables, mostró que no hubo mejoría en la tasa de ovulación con $1500 \mathrm{mg}$ diarios de metformina, a pesar de la reducción en el índice de masa corporal y los niveles de testosterona.

Vandermolen y otros estudiaron en 27 mujeres si la metformina asociada con citrato de clomifeno aumentaba las tasas de ovulación y embarazo. La investigación aleatoria por computadora, doble ciego y controlada con placebo, demostró que 9 de 12 en el grupo de estudio ovularon (75\%), versus 4 de 15 en el de control (27\%). Hubo fertilidad en 6 de 11 del grupo tratado con metformina $1500 \mathrm{mg}$ por día, asociado con $100 \mathrm{mg}$ por día de citrato de clomifeno (55\%), y 1 de $14(7 \%)$ en el grupo placebo. ${ }^{30}$

Sturrock y colaboradores realizan un estudio aleatorio cruzado, controlado con placebo, doble enmascarado, de 26 pacientes resistentes al citrato de clomifeno. Doce recibieron metformina por 3 meses, $1500 \mathrm{mg}$ por día, seguida por metformina con citrato de clomifeno por 3 ciclos, y 14 actuaron como control. Su objetivo consistía en valorar la restauración de la ovulación y la tasa de fertilidad. ${ }^{31} \mathrm{La}$ ovulación espontánea se reanuda en 5 pacientes tratadas con metformina, versus 6 en el grupo placebo. No hubo diferencia en cuanto a la ovulación en ambos grupos. El embarazo ocurrió en 3 de las pacientes del grupo de estudio, versus 2 del grupo control. No se mencionan los riesgos relativos de cada grupo ni el grado de precisión del efecto del tratamiento. El estudio concluye que no todas las 
pacientes con SOP, resistentes al citrato de clomifeno, se benefician del tratamiento con metformina.

Heard y otros analizan una serie de 48 pacientes anovulatorias durante un periodo de 15 meses. Todas fueron iniciadas con metformina $1000 \mathrm{mg}$ por día, por 6 semanas. Si no se producía ovulación, la dosis se incrementaba a 1500 mg por día, por 6 semanas. De persistir la falta de respuesta ovulatoria se agregaba citrato de clomifeno. ${ }^{32}$ Diecinueve pacientes del total estudiado reanudan las ovulaciones espontáneas solo con metformina (40\%), lo que se documenta por temperatura basal y monitoreo de hormona luteinizante, mientras que un $31 \%$ requirió asociación con citrato de clomifeno.

Nestler y colaboradores refieren que es necesario un estudio aleatorio y prospectivo, de metformina versus clomifeno como medicamento inicial para inducir la ovulación en pacientes con SOP, con el propósito de decidir sobre esta práctica clínica. ${ }^{33}$

Costello y Eden, en su revisión sistemática, señalan que 9 estudios evalúan la efectividad de la metformina sola en restaurar la ovulación en pacientes no seleccionados con SOP. Siete de las investigaciones comprometen un número pequeño de pacientes $(<50)$ con una duración de 1 a 26 meses de tratamiento. Los datos combinados de 5 estudios observacionales y no controlados muestran que el $61 \%$ de las mujeres con SOP ovulan con solo metformina. ${ }^{17}$ Cuatro estudios aleatorios comparan la metformina con el placebo. El 56\% de los pacientes ovulan en comparación con el $35 \%$ con placebo, con un RR de 1.5 y un rango de confianza al $95 \%$, de 1.2 a 2.0 .

Los mismos autores identifican 5 estudios que valoran la efectividad de la metformina asociada con citrato de clomifeno, en la restauración de la ovulación y embarazo. Combinando los resultados de las 3 investigaciones observacionales, un $66 \%$ de las pacientes ovulan y un $34 \%$ logran fertilidad, después de tratamiento asociado mayor de 9 meses. Los investigadores concluyen, con 2 estudios aleatorios, que la asociación de metformina con citrato de clomifeno incrementa la respuesta ovárica en mujeres obesas con SOP, así como la tasa de fertilidad. En pacientes resistentes al citrato de clomifeno, la metformina asociada mejoró la ovulación (RR 4, con intervalo de confianza al $95 \%$, de 1.6 a 4.1 ) y la tasa de fertilidad (RR 2.2 con intervalo de confianza al $95 \%$, de 1.5 a 3.0$).{ }^{17}$

McCarthy y otros indican que 8 estudios aleatorios muestran que la ovulación ocurrió en 77 de las 184 pacientes tratadas con solo metformina $(42 \%)$ y 38 de 184 casos tratados con placebo $(22 \%)$. La asociación de citrato de clomifeno aumenta la ovulación en 61 de 91 casos tratados (66\%), versus 13 de 91 controles (16\%). Cuatro estudios aleatorios reportan 12 concepciones en 94 pacientes tratadas con metformina (13\%) y 4 en 94 controles (4.2). ${ }^{26}$

Lord y Wilkin se refieren a la revisión Cochrane y encuentran un beneficio significativo con el uso de metformina asociada con citrato de clomifeno, versus solo citrato de clomifeno en las tasas de ovulación (OR 4.41, límite de confianza al 95\%, de 2.37 a 8.22) y de embarazo (OR 4.40, rango de confianza al 95\%, de 1.96 a 9.85), siendo 3 el número de pacientes necesarias de tratar. ${ }^{34}$

Kashyap y colaboradores señalan que la metformina es un 50\% mejor que el placebo al inducir la ovulación en mujeres infértiles con SOP (RR 1.50, rango de confianza al $95 \%$, de 1.13 a 1.99$).{ }^{19}$ Sin embargo, no se ha confirmado beneficio para el logro de embarazo (RR 1.07, con límite de confianza al $95 \%$, de 0.20 a 5.74). La metformina asociada al citrato de clomifeno es superior al clomifeno solo, en cuanto a ovulación (RR 3.04, con rango de confianza al $95 \%$, de 1.77 a 5.24) y logro de fertilidad (RR 3.65, con limite de confianza al 95\%, de 1.11 a 11.99).

Otra revisión refiere que la metformina sola induce más la ovulación que el placebo (OR 3.88; intervalo de confianza al 95\% 2.55 a 6.69). La metformina asociada con el citrato de clomifeno es más efectiva que el clomifeno en inducir la ovulación (OR 4.41; intervalo de confianza de 2.37 a 8.22). Las mujeres con SOP resistentes al clomifeno y con un IMC mayor de $25 \mathrm{Kg}$. $/ \mathrm{m}^{2}$, no muestran aumento en la tasa de embarazos clínicos cuando la metformina es usada como monoterapia. Pero el tratamiento asociado, metformina y citrato de clomifeno, incrementó la tasa de embarazo clínico (OR 4.88; intervalo de confianza al 95\% 2.37 a 8.22). ${ }^{35}$

Palomba y colaboradores publican un estudio aleatorio, controlado con placebo y doble ciego, de 100 mujeres no obesas con SOP. Un grupo recibió $850 \mathrm{mg}$ bid de metformina más placebo por 6 meses, y el otro, placebo más $150 \mathrm{mg}$ de citrato de clomifeno por 5 días, a partir del tercer día del ciclo. Los datos fueron de 45 mujeres (221 ciclos) en el grupo A y 47 (205 ciclos) en el B. Aunque la tasa de ovulación fue similar en ambos grupos (62.9\% versus $67 \%)$, la de fertilidad fue mayor con metformina ( $15.1 \%$ versus $7.2 \%){ }^{36}$

\section{Conclusiones}

Se podría concluir que la metformina es beneficiosa para regular los ciclos menstruales, en comparación con el placebo que su uso como tratamiento contra el hirsutismo no debe ser considerado de primera elección. La práctica del uso de metformina como monoterapia en la inducción de ovulación no puede recomendarse para uso rutinario, hasta que se publiquen datos de grupos mayores de pacientes y se indique un mejor efecto en la tasa de fertilidad. Aunque se ha demostrado que incrementa la ovulación, no mejora la tasa de embarazo clínico. Existe evidencia sustancial de que la metformina asociada con el citrato de clomifeno induce la ovulación. Existe evidencia sustancial de que la metformina 
asociada con el citrato de clomifeno mejora la tasa de embarazo clínico.

\section{Abstract}

Metformin has been prescribed for treating type II Diabetes Mellitus of patients with normal renal function. It has also been used with the FDA approval for patients with polycystic ovary syndrome as regulator of the menstrual cycle, and for the treatment of hirsutism and as an ovulation inductor.

Since 1980, Burgen et al. pointed the association between hyperandrogenism and hyperinsulinism in patients with polycystic ovary syndrome. The excess of androgens due to the increase of insulin diminish its action.

Metformin mechanism for lowering glucose is a consequence of a decrease in hepatic glucose production and an increase of its utilization. Metformin increases most of insulin biological actions in people with pre-existing insulin resistance and decreases intestinal glucose absorption.

In conclusion, metformin is useful for menstrual cycle regulation in comparison with placebo, is not a first choice treatment for hirsutism, and its use as monotherapy for induction of ovulation has not demonstrated a substantial rate improvement in clinical pregnancy. Evidence shows that metformin associated with clomiphene citrate induces ovulation and improves clinical pregnancy rate.

\section{Referencias}

1. Kirpichnifov D, McFarlane S, Sowers J. Merformin: An Update. Ann Intern Med 2002; 137: 25-33

2. Burghen G, Givens J, Kitabchi A. Correlation of hyperandrogenism with hyperinsulinism in polycystic ovarian disease. J Clin Endocrinol Metab 1980; 50:113-116

3. Dunaif A, Segal KR, Green G, Dobrjansky A, Licholai T. Evidence for distinctive and intrinsic defects in insulin action in the polycystic ovary syndrome. Diabetes 1992; 41:1257-1266

4. Holte J, Bergh T, Berne C, Berglund L, Lithell H. Enhanced early insulin response to glucose in relation to insulin resistance in women with polycystic ovary syndrome. J Clin Endocrinol Metab 1994; 78: 1052-1058

5. Cibula D. Is insulin resistance an essential component of PCOS? Hum Reprod 2004; 19:757-759

6. Barbieri R. Induction of ovulation in infertile women with hyperandrogenism and insulin resistance. Am J Obstet Gynecol 2000; 183:1414-1418

7. The Rotterdam ESHRE/ASRM-Sponsored PCOS consensus workshop group. Revised 2003 consensus on diagnostic criteria and long-term health risks related to polycystic ovary syndrome. Fertil Steril 2004; $81: 19-25$
8. Alberti J, Zimmet P, Shaw J. The metabolic syndrome-a new worldwide definition. Lancet 2005; 366: 1059-1062

9. Elkind-Hirsh K, Chang J. Use of insulin sensitizing agent in the treatment of polycystic ovary syndrome. American Society for Reproductive Medicine. Committee Opinion April 2000

10. Bailey C, Path M, Turner R. Metformin. N Engl J Med 1996; 334: 574-579

11. Moghetti P. Insulin resistance: what is its role in the polycystic ovary syndrome? Curr Opin Endocrinol Diabetes 2002; 9:444-450

12. Barbieri, R. Metformin for treatment of the polycystic ovary syndrome. UpToDate, Rose, BD(Ed), UpToDate, Wellesley, MA, 2004. Recuperado el 10 de enero del 2004 de http:// www.uptodate.com

13. Metformin: Drug Information. UpToDate, Rose, BD(Ed), UpToDate, Wellesley, MA, 2004. Recuperado el 10 de enero de 2004 de http:// www.uptodate.com

14. Misbin R, Stadel B. Lactic acidosis in patients with diabetes treated with metformin. N Engl J Med 1998; 338: 265 -266

15. Lalau JD, Race JM. Lactic Acidosis in Metformin Therapy. Drugs 1999; 58 Suppl.1: 55 - 60

16. Calabrese A, Coley K, DaPos S, Swanson D, Rao R. Risk of Lactic Acidosis with Metformin Therapy. Arch Intern Med 2002; 162: 434437

17. Costello MF, Eden JA. A systematic review of the reproductive system effects of metformin in patients with polycystic ovary syndrome. Fertil Steril 2003; 79: 1-13

18. Harborne L, Fleming R, Lyall H, Norman J, Sattar N. Descriptive review of the evidence for the use of metformin in polycystic ovary syndrome. Lancet 2003;361: 1894 - 1901

19. Kashyap S, Wells G, Rosenwaks Z. Insulin - sensitizing agents as primary therapy for patients with polycystic ovary syndrome. Hum Reprod 2004; 19: 2474-2483

20. Crave JC, Fimbel S, Lejeune H, Cugnardey N, Dèchaud H, Pugeat M. Effects of diet and metformin administration on sex hormone-binding globulin, androgens and insulin in hirsute and obese women. J Clin Endocrinol Metab 1995; 80:2057-2062

21. Kolodziejczyk B, Duleba A, Spaczynski R, Pawelczyk L. Metformin therapy decreases hyperandrogenism and hyperinsulinemia in women with polycystic ovary syndrome. Fertil Steril 2000; 73:1149-1154

22. Elter K, Imir G, Durmusoglu F. Clinical, endocrine and metabolic effects of metformin added to ethinyl estradiol-cyproterone acetate in non-obese women with polycystic ovarian syndrome: a randomized controlled study. Hum Reprod 2002; 17: 1729-1737

23. Harborne L, Fleming R, Lyall H, Sattar N, Norman J. Metformin or antiandrogen in the treatment of hirsutism in polycystic ovary syndrome. J Clin Endocrinol Metab 2003; 88: 4116-4123

24. Rosa Maciel G, Soares J, Alves E, Haidar M, Rodríguez E, Baracat E. Nonobese women with polycystic ovary syndrome respond better than obese women to treatment with metformin. Fertil Steril 2004; $81: 355-360$

25. Cibula D. Fanta M, Vrbikova J, Stanicka S, Dvorakova K, Hill M. The effect of combination therapy with metformin and combined oral contraceptives versus COC alone on insulin sensitivity, hiperandrogenaemia, SHBG and lipids in PCOS patients. Hum Reprod 2004; 20:180-184

26. McCarthy E, Walker S, McLachlan K, Boyle J, Permezel M. Metformin in Obstetrics and Gynecologic Practice: a Review. Obstet Gynecol Survey 2004; 59: 118-127

27. Nestler J, Jakubowics J, Evans W, Pasquali R. Effects of metformin on spontaneous and clomiphene - induced ovulation en the polycystic ovary syndrome. N Engl J Med 1998; 338:1876-1780

28. Batukan C, Baysal B. Metformin improves ovulation and pregnancy rates in patients with polycystic ovary syndrome. Arch Gynecol Obstet 2001; 265: 124-127 
29. Hung E, Ming N, Chung P. Effects of metformin on ovulation rate, hormonal and metabolic profiles in women with clomiphene-resistant polycystic ovaries: a randomized, double-blinded placebo-controlled trial. Hum Reprod 2001; 16:1625-1631

30. Vandermolen D, Ratts V, Evans W, Stovall D, Kauma S, Nestler J. Metformin increases de ovulatory rate and pregnancy rate from clomiphene citrate in patients with polycystic ovary syndrome who are resistant to clomiphene citrate alone. Fertil Steril 2001; 75: 310-315

31. Sturrock N, Lannon B, Fay T. Metformin does not enhance ovulation induction in clomiphene resistant polycystic ovary syndrome in clinical practice. Br J Clin Pharmacol 2002; 53:469-473

32. Heard M, Pierce A, Carson S, Buster J. Pregnancies following use of metformin for ovulation induction in patients with polycystic ovary syndrome. Fertil Steril 2002; 77; 669-673
33. Nestler J, Stovall D, Akhter N, Iuorno M, Jakubowics D. Strategies for the use of insulin-sensitizing drugs to treat infertility in women with polycystic ovary syndrome. Fertil Steril 2002; 77:209-215

34. Lord J, Wilkin T. Metformin in polycystic ovary syndrome. Curr Opin Obstet Gynecol 2004; 16: 481-486

35. National Collaborating Centre for Woman's and Children Health. Fertility: assessment and treatment for people with fertility problems. Ovulation induction 2004. Recuperado el 1 de junio del 2005 de http:// www.nice.org.uk/pdf/CG011fullguideline.pdf

36. Palomba S, Orio F, Falbo A, Manguso F, Russo T, Cascella T. Prospective parallel randomized double-blind double-dummy controlled clinical trial comparing climifene citrate and metformin as the first-line treatment for ovulation induction in non-obese anovulatory women with polycystic ovary syndrome. J Clin Endocrinol Metab 2005; 90:4068 - 4074 